ISSN 2447-9071

doi https://doi.org/10.36414/rbmc. v5i13.25

Contato para correspondência: Antonio Márcio Teodoro Cordeiro Silva

E-mail:

marciocmed@gmail.com

Conflito de interesse: Não

Financiamento: Recursos próprios

Recebido: 20/11/2019

Aprovado: 11/12/2019

\section{A tecnologia de CRISPR-Cas9 na terapia gênica do câncer de pulmão}

\section{CRISPR-Cas9 technology in lung cancer gene therapy}

Raphael Ladislau de Alcantara', Hellen Patrícia Santos Lima', Gabriel de Oliveira Barbosa', Lucas Cândido Gonçalves ${ }^{1}$, Adriana Priscila Lázaro da Silva' ${ }^{1}$ Xisto Sena Passos ${ }^{1}$, Antonio Márcio Teodoro Cordeiro Silva ${ }^{1,2,3}$

1 Universidade Paulista - UNIP

${ }^{2}$ Pontifícia Universidade Católica de Goiás - PUC Goiás

${ }^{3}$ Faculdade da Polícia Militar - FPM

\section{Resumo}

O presente estudo objetivou avaliar a possibilidade do CRISPR-Cas9 na terapia gênica do câncer de pulmão, por meio do conhecimento do mecanismo da CRISPR-Cas e da sua utilização no tratamento do câncer de pulmão. Trata-se de uma revisão de literatura de aspecto descritivo. Foram excluídas as publicações e artigos repetidos que não eram relacionadas ao tema, totalizando 22 referências e tendo como 41 referências que constituem o presente trabalho, retiradas no site da National Center for Biotecnology Information (NCBI), na base de dados PubMed. Foram utilizados os descritores: CRISPR/Cas9, Lung Cancer, Gene therapy, gene edition, adaptive immunity, após foram catalogadas esubmetidas a análise. Estudos de artigos referentes àterapia gênica, através da utilização do CRISPRCas9, demonstraram diversas possibilidades de utilização do sistema para o tratamento de tumores, indicando um possível tratamento do câncer de pulmão pelo sistema CRISPR-Cas9, dependendo somente de pesquisa e estudos. O sistema CRISPR-Cas9 pode ser considerado a tecnologia do século, por proporcionar uma plataforma adequada para o desenvolvimento de pesquisas relacionadas aos adenocarcinomas em condições in vivo e in vitro e trazer diversas possibilidades para tratar o câncer de pulmão com base na terapia gênica.

Palavras-Chave: CRISPR-Cas9, Terapia gênica, Câncer de pulmão.

\section{Abstract}

The present study aimed to evaluate the possibility of CRISPR-Cas9 in gene therapy of lung cancer by knowing the mechanism of CRISPR-Cas and its use in the treatment of lung cancer. This is a descriptive literature review. Repetitions and publications that were notrelated to the theme wereexcluded, totaling 22 references and having as 41 references that constitute the present work, taken from the National Center for Biotechnology Information (NCBI) website, in the PubMed database. Using the descriptors CRISPR/Cas9, Lung Cancer, Gene therapy, afterwere cataloged and analyzed. Studies of articles related to gene therapy using CRISPR-Cas9 showed several possibilities of using the system to treat tumors, indicating a possible treatment of lung cancer by the CRISPR-Cas9system, depending only on research and studies. The CRISPR-Cas9system can be considered the technology of the century, as itprovides an adequate platform for the development of adenocarcinoma research in vivo and in vitro conditions and brings several possibilities to treat lung cancer based on gene therapy.

Keywords: CRISPR-Cas9, Gene therapy, Lung cancer. 


\section{Introdução}

O câncer de pulmão, por ser o tumor mais comum no mundo, é uma das principais causas de morte, com cerca de 1,8 milhão de diagnósticos e 1,6 milhão de óbitos' ${ }^{1}$. Pacientes com câncer de pulmão apresentam grande risco de desenvolver um segundo tumor e os seus descendentes estão mais propícios a adquirir tal enfermidade, devido aos fatores hereditários ${ }^{2}$. O tabagismo é a principal causa de câncer de pulmão, sendo responsável por, aproximadamente, sete milhões de mortes anuais no mundo, incluindo o câncer ${ }^{3}$.

Repetições palindrômicas curtas agrupadas e interespaçadas (CRISPR, do inglês, Clustered regulary interspaced short palindromic repeats) e genes que codificam proteínas associadas (Cas, do inglês, associated proteins) são uma nova técnica utilizada atualmente para promover alteração gênica e surgem como nova alternativa para nucleases com efetores do tipo ativador transcricional (TALEN, do inglês, transcription activator-like effector nucleases) e nuclease dedo de zinco (ZFN, do inglês, Zine-finger nucleases), técnicas estas, utilizadas para edição genômica ${ }^{4,5}$. Foi descoberta por volta da década de 1980, na bactéria Escherichia coli ${ }^{6}$, que utilizava a proteína como um mecanismo de defesa contra vírus e plasmídeos ${ }^{7}$. O sistema imunológico da bactéria reconhece o gene do vírus, corta e insere em seu próprio DNA, pois, em caso de uma infecção futura, o CRISPR/Cas entra em ação, que vão reconhecer os ácidos nucleicos do gene do vírus e destruí-lo, fazendo a quebra de parte do gene conhecido 8 . O CRISPR/Cas é classificado em 3 tipos: I, II e lllI9. E foi o tipo II, CRISPR/Cas ${ }^{9}$, que demonstrou maior facilidade de edição do genoma ${ }^{10}$.

A terapia gênica surgiu com intuito de tratar doenças genéticas, e logo foi observado uma nova metodologia para o tratamento contra o câncer, criando, com isso, vários vetores virais e não virais para fazer alteração no DNA da célula ${ }^{11}$. Alguns fatores dificultam e limitam a terapia gênica como: não ter alta seletividade e baixa eficiência de transferência. Quesitos importantes em doenças como o câncer, cujo tratamento seria eficiente, tratando-se com uma alta seletividade e eficiência de transferência, o que torna o tratamento decisivo ${ }^{12}$.

A ideia de utilizar o CRISPR surgiu em 2012, como uma nova técnica de edição genômica, utilizando da terapia gênica, uma metodologia da engenharia genética, capaz de fazer reparação em genes alterados, modificações pontuais, técnica buscada pela medicina desde o entendimento do DNA ${ }^{13,14}$. Esta pesquisa objetivou estudar o CRISPR-Cas9, no contexto da terapia gênica aplicada ao câncer de pulmão através de uma revisão de literatura.

\section{Métodos}

Este estudo constituiu-se de revisão da literatura sobre o CRISPR-Cas9 na terapia gênica do câncer de pulmão. Foram então acessados, no período de junho a setembro de 2019, nos bancos de dados da Literatura Latino-Americana e do Caribe em Ciências da Saúde (LILACS), Scientific Electronic Library Online (SciELO), e no site da National Center for Biotecnology Information (NCBI), na base de dados PubMed, utilizando-se dos mesmos descritores. A busca das referências, em língua portuguesa, foi realizada após a consulta aos Descritores em Ciências da Saúde (DeCS): CRISPER-Cas9, Câncer de pulmão, Terapia gênica, Edição gênica, Imunidade adaptativa. E para a busca das referências, em língua inglesa, foi acessado o Medical Subject Headings (MeSH): CRISPR-Cas9, Lung Cancer, Gene therapy, Gene edition, Adaptive immunity, utilizando dos booleanos "AND" e "OR".

No LILACS, foram encontradas uma referência, no SciELO, três referências e na PubMed 60 referências. Todas com disponibilidade de título, ano, local da publicação e resumo. Foram adotados como critérios de inclusão, artigos originais que discorriam sobre a utilização de CRISPR-Cas9 em cânceres e o seu sistema de funcionamento. Como critério de exclusão as repetições e as publicações que não estavam relacionadas ao tema após a leitura dos resumos, que não abordavam a utilização do CRISPR-Cas9 em cânceres ou seu sistema de funcionamento, resultando em 22 referências excluídas.

As 42 referências restantes, que constituíram esta amostra, foram, primeiramente, catalogadas e analisadas. Os artigos científicos foram analisados e algumas dificuldades encontradas para a coleta de dados foram o fato de alguns resumos não conterem informações sobre as abordagens metodológicas e objetivos. Apesar dessas dificuldades encontradas em algumas produções, foi possível obter as informações desejadas nos estudos.

\section{Resultados}

\section{Conhecendo o CRISPR-Cas}

O CRISPR-Cas, descoberto em bactéria e archea, sendo o primeiro e o único sistema mediado por RNA de imunidade adaptativa conhecido. Esse sistema de defesa depende de pequenos RNAs para detectar as sequências específicas e com isso efetuar o silenciamento de ácidos nucleicos estranhos. Os genes Cas são responsáveis por codificar as proteínas funcionais conhecidas como complexos efetores. A diversidade de componentes encontrados no sistema CRISPR-Cas é impressionante, mas por possuir vários elementos de assinatura em sua organização genômica, forneceu uma classificação simples para esse sistema. O CRISPR-Cas é dividido em duas classes e cada 
classe possui vários tipos. Geralmente, a classe 2 e mais simples que a classe 115 (Figura 1). Os complexos efetores do sistema de classe 1 compreendem de várias proteínas Cas. Já a classe 2 apresenta uma proteína grande que possui vários domínios ${ }^{16}$.

Tal classificação é dividida em três tipos cada, na classe 1 os tipos I, III e IV, na classe 2, os tipos II, V e VI. Cada tipo possui características de arquitetura diferente dos módulos efetores que possuem proteínas de assinatura exclusivas. Além disso, cada tipo é classificado em múltiplos subtipos que se tornam diferenciados pela sutil divergência na organização do locus e pela frequência de codificação de proteínas Cas específica por subtipo ${ }^{17}$.

Os sistemas I e III são parecidos, sendo que ambas as suas interferências envolvem crRNP (complexos de proteínas ribonucleicas), onde cada um é composto por um crRNA guia e um complexo de proteínas Cas. Já o tipo ll é específico para um crRNA duplo com um RNA adicional (tracrRNA) formando um complexo de DNA que interfere com uma única proteína, a Cas9 ${ }^{18}$.

O sistema CRISPR-Cas tipo II é mais simples em termos de número de genes em relação ao tipo I e III. A Cas9 é o gene de assinatura do tipo II que codifica uma proteína de múltiplos domínios que associa-se às funções do complexo efetor de crRNA com o corte do DNA-alvo e contribui com a adaptação ${ }^{19}$.

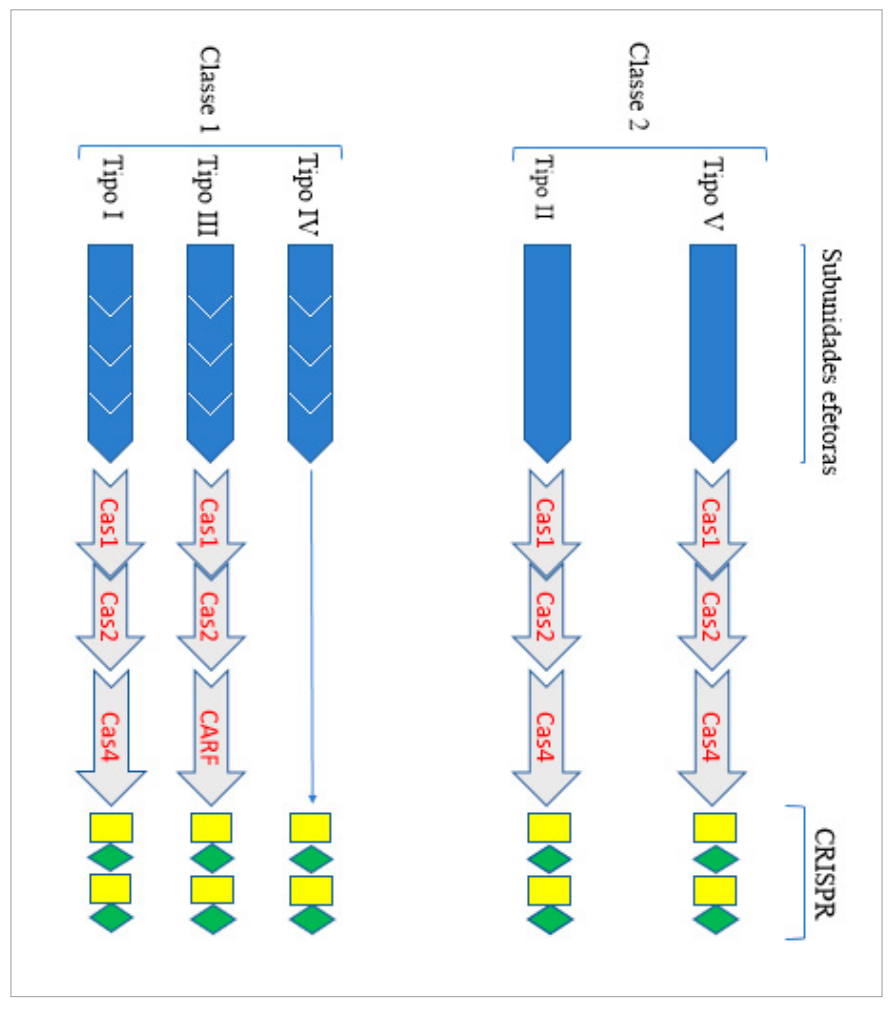

Figura 1: Sistema de classes e tipos do CRISPR (adaptado de Karimian, 2019).
A maioria dos loci do tipo Il codifica o tracrRNA que é parcialmente complementar às repetições dentro da matriz CRISPR respectiva. Também todos os loci identificados do tipo II possuem a Cas1 e a Cas2. Portanto, a presença de Cas9 próximo a Cas1 e os genes da Cas2 são a marca registrada do loci tipo II $^{16}$.

\section{Mecanismo de atuação da tecnologia CRISPR-Cas}

Quando em contato com um DNA estranho o CRISPR-Cas atua em três etapas, a adaptação (aquisição do espaçador), expressão (o processo de crRNA) e interferência (destruição do material genético invasor) ${ }^{9}$.

Na fase de adaptação, um pequeno segmento de DNA invasor deve ser integrado no cromossomo hospedeiro. Nesta fase, um segmento especial (protoespaçador) é separado do DNA invasor (bacteriófago ou plasmídeo) e inserido e integrado na extremidade $5^{\prime}$ de uma matriz CRISPR, através do complexo de proteínas Cas1-Cas2. Este segmento inserido faz de uma unidade espaçadora nova especial ${ }^{15}$.

Na segunda etapa, irá criar o crRNA. Com isso, nesse estágio de expressão, um CRISPR array (repetições e espaçadores) será transcrito para um RNA longo (pré-crRNA) que será clivado e processado em RNAs curtos (crRNAs). Para converter o précrRNA em crRNAs é necessário um complexo efetor de crRNA multisubunidade ou Cas9. A extremidade da cadeia $5^{\prime}$ do crRNA é de cadeia simples e determina o grau de ligação das sequências-alvo ${ }^{16}$.

Na terceira etapa, de interferência, o crRNA-Cas9 é capaz de clivar o material genético estranho ou o material genético alvo. O protoespaçador adjacente é importante para reconhecer os alvos do Cas 9 ou dos outros complexos Cas. Após a identificação da sequência-alvo, ocorre o emparelhamento de bases entre o alvo invasor e o crRNA iniciando então a atividade da nucleasse $^{20}$. O resumo das três etapas está esquematizado na Figura 2.

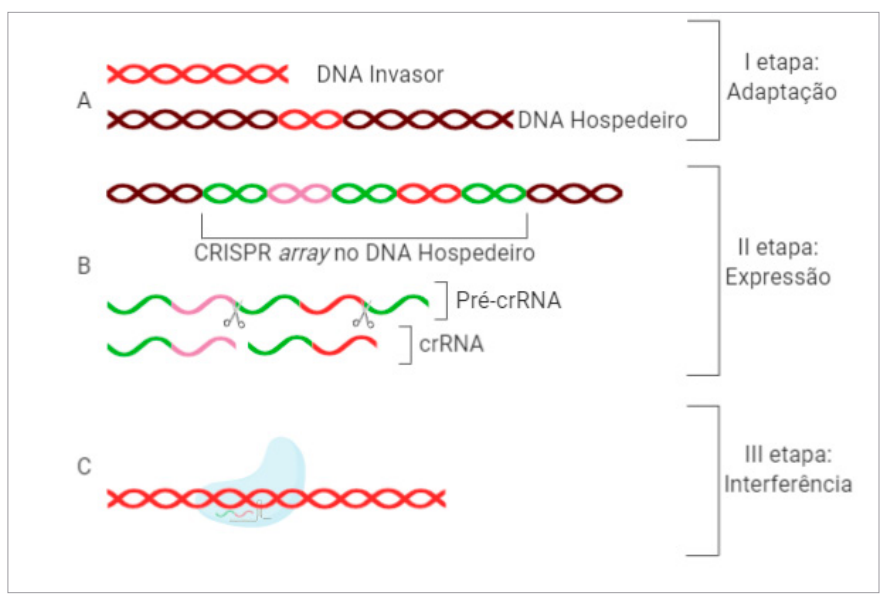

Figura 2: As etapas da tecnologia CRISPR. 


\section{CRISPR-Cas e seu desenvolvimento na terapia gênica}

A engenharia genética vem manipulando DNA e RNA para promover formas de prevenção e tratamento de doenças em humanos ou em animais ${ }^{21}$. Essa manipulação está envolvida com técnicas de manipulação de genes, como deleção, substituição ou inserção, criando uma relação com os distúrbios genéticos. As principais ferramentas utilizadas para essas técnicas são: TALENs, ZFNs e, a mais recente e com maiores perspectivas, o sistema CRISPR-Cas ${ }^{22}$.

O DNA double-strand breaks (DSBs) poderia estimular bastante a edição do genoma por meio de eventos de recombinação mediados por recombinação homóloga. E para conseguir uma eficaz edição do genoma por meio do DSBs de DNA específico, projetou quatro classes principais de proteínas de ligação ao DNA, a ZFNs e a TALENs entre elas, reconhecendo sequência de DNA através de interações proteína-DNA ${ }^{23}$. No sistema CRISPR, as sequências-alvo se ligam ao RNA guia único (sgRNA, single guide RNA). Com isso o sistema CRISPR se torna mais aplicável que os demais sistemas e possui vários diferenciais importantes. Por exemplo, a demanda da mão de obra que ZFNs eTALENs necessitam quando comparados com a tecnologia CRISPR. Além disso, oCRISPR-Cas tem potencial para edição multiplexada simultânea de genes em comparação com ZFNs e TALENs ${ }^{24}$.

O CRISPR-Cas tem sido utilizado para terapia de câncer em testes in vivo e in vitro. E com o passar dos anos e com as novas tecnologias, essa ferramenta passou a ser utilizada com objetivos terapêuticos, principalmente em doenças infecciosas $^{25}$. Outra maneira da utilização da técnica está relacionada a distúrbios genéticos ${ }^{15}$.

\section{CRISPR-Cas9 no tratamento do câncer de pulmão}

O câncer de pulmão, quando é primário, possui uma abordagem terapêutica diferente de quando se trata de metástase para os pulmões, quando o câncer inicia em outra parte do corpo e se espalha para o pulmão. Quanto ao aspecto histológico, existem dois grandes grupos desta patologia: câncer de pulmão de pequenas células (CPPC) e câncer de pulmão de não pequenas células (CPNPC), dependendo do tipo de célula em que o câncer se inicia ${ }^{26}$.

O diagnóstico do câncer de pulmão, comumente, apresenta dificuldades que interferem na sobrevida do paciente. Na maioria dos casos, o diagnóstico de CPNPC costuma ocorrer em estágios avançados, o que dificulta o tratamento ${ }^{27}$. E cerca de $14 \%$ de todos os novos diagnósticos de câncer se revela como câncer de pulmão, em que o CPNPC costuma ser o tipo dominante, representando 80 a $85 \%$ de todos os diagnósticos de câncer de pulmão. Mesmo sendo, o de pior prognóstico, o CPPC, é responsável por 10 a $15 \%$ dos cânceres de pulmão ${ }^{28}$.

Atualmente, o tratamento do câncer de pulmão depende do seu estágio e da sua categoria, por exemplo: pacientes em estágio inicial de CPNPC, a cirurgia é o tratamento recomendado $^{29}$. E em estágio mais avançados, já é indicado terapia com fármacos específicos para anormalidades moleculares ${ }^{30}$. Existem três alternativas para se tratar o câncer de pulmão: a cirurgia, a radioterapia e a quimioterapia ${ }^{31}$.

Geralmente, defeitos genéticos ou mutações em células tumorais possuem características específicas. Com isso, silenciar a expressão de proteínas específicas ou reparar genomas de tumores se torna importante para a pesquisa e para o tratamento de tumores ${ }^{32}$. O sistema de edição de genes CRISPR-Cas9, por possuir vantagens como execução simples, alta eficiência e especificidade, pode ser utilizado para editar genomas, eliminando genes específicos ou, nos tumores, reparando a mutação $0^{33}$.

Devido os tumores possuírem várias mutações genéticas, o CRISPR-Cas9 pode ser utilizado para construir um modelo de tumor com múltiplas mutações no gene in vivo e com isso simular doenças complexas ${ }^{34}$. Em um estudo a deleção de AMPK a1 e a2, através de uma edição feita com CRISPR-Cas9 em células de adenocarcinoma de pulmão (CPNPC mediadas em KRASG12D p53f/f), demonstrou uma diminuição substancial no tamanho do tumor ${ }^{35}$. O CRISPR-Cas9 também foi utilizado para obter mutações comuns nos genes Kras, P53 e Lkb1, em camundongos, levando a alterações patológicas do adenocarcinoma pulmonar em camundongos ${ }^{36}$.

Até o final de 2018, apenas um ensaio clínico foi implementado, utilizando CRISPR-Cas9 como uma maneira de intervenção para o câncer de pulmão. Esse estudo foi realizado pela Universidade de Sichuan, que avaliou a segurança das células T modificadas para inibição de PD-1 no tratamento do CPPC metastático (ID do ensaio: NCT02793856) ${ }^{26}$.

O CRISPR-Cas9 é uma ferramenta de edição poderosa e com um grande potencial ${ }^{37}$. Devido potencial, poucos projetos conseguem aprovações éticas para conduzir testes clínicos em humanos, principalmente, testes in vitro para edição de genomas das células ${ }^{38}$.

\section{Discussão}

Segundo Nair et al. ${ }^{26}$, as técnicas com CRISPR têm grande potencial de trazer avanços na pesquisa com câncer. Isso se dá devido à grande facilidade de execução em comparação com as técnicas existentes, como ZNFs e TALENs, além do fato da sua especificidade e eficiência serem altas. Seu potencial proporciona grandes avanços para a medicina em diversas áreas da saúde, como demonstra Jiang et al..$^{34}$ que indicam uma perspectiva de criação de medicamentos.

O sistema CRISPR-Cas é um sistema que se tornou complexo devido a uma série de eventos evolutivos, para que houvesse 
adaptação da imunidade microbiana, o que torna sua classificação confusa, principalmente por possuir e ainda estarem descobrindo novos subtipos. De acordo com Makarova et al. ${ }^{39}$, tal classificação pode ser melhorada, tornando o sistema mais consistente e esta tarefa pode ser realizada dentro de alguns anos.

Knigth et al. ${ }^{40}$ concluíram que cerca de $75 \%$ dos diagnósticos de câncer de pulmão estão em estágios avançados e que $70 \mathrm{a}$ $90 \%$ dos diagnósticos em estágio inicial podem garantir melhor sobrevida ao paciente. Por não possuir sinais específicos e ter os sintomas parecidos para outras doenças respiratórias, o diagnóstico precoce do câncer de pulmão se torna difícil, pelo fato dos sinais e sintomas surgirem em estágios mais avançados da doença, além da questão do tipo de tumor em CPPC e CPNPC que possuem sobrevidas e tratamento diferentes, dependendo do estágio que estiverem.

O CRISPR-Cas9 se tornou a ferramenta de edição genética mais utilizada, apresentando grandes perspectivas para a terapia gênica. Sua utilização, associada a outras técnicas de edição, torna da terapia genética um possível tratamento de inúmeras patologias de maneiras diversificadas. Segundo Xiao-Jie et al. ${ }^{25}$, um ponto importante da terapia gênica em câncer é as modificações de células hospedeiras, melhorando a resposta imune ou aumentando a resistência das células. Modificações em células de defesa, como em células $T$, são realizadas in vitro e reimplantadas nos pacientes após terem sido modificadas geneticamente, fornecendo uma aplicação a terapia imune através do CRISPR-Cas9.

Outras maneiras possíveis de utilizar o CRISPR-Cas9 é por meio da edição de genes supressores de tumores ou em oncogenes. $\mathrm{O}$ estudo de Moses et al. ${ }^{41}$ demostrou a capacidade do sistema CRISPR-Cas de realizar a ativação da PTEN, uma proteína supressora de tumor essencial, que quando silenciada, promove comportamento metastático e resistência à terapia de cânceres altamente agressivos. Diante de tantas possibilidades, a utilização desse sistema tão complexo traz grandes perspectivas para a terapia genética.

A utilização de CRISPR-Cas9 pode vir a ser inevitável, devido ao desenvolvimento humano, porém envolve questões éticas, principalmente a sua possibilidade de manipular geneticamente organismos vivos. Segundo Oliveira e Reis ${ }^{42}$, questões éticas ainda são debatidas no mundo todo diante da utilização do sistema CRISPR-Cas9 com diferentes objetivos e organismos. Levantam questões de biossegurança e bioéticas referentes a Lei brasileira n. 11.105/2005, em que pesquisas e terapias com embriões humanos são permitidos apenas com células-tronco, sendo proibida qualquer técnica de engenharia genética, como a técnica de CRISPR-Cas9. A utilização dos embriões devem seguir requisitos: ter por alvo as células-tronco embrionárias, genitores consentir a utilização, aprovação do comitê de ética e não destinar a comercialização de material biológico.

\section{Conclusão}

O CRISPR pode ser considerado a tecnologia do século, por possuir diferentes campos de atuação, eficiência e sensibilidade superiores às técnicas já existentes com possibilidade de aplicação em terapia gênica. O número de tratamentos para o câncer de pulmão ainda é baixo e a utilização dessa técnica no câncer de pulmão e em outros tumores pode fazer total diferença no tratamento dessas patologias, além de proporcionar plataforma adequada para o desenvolvimento de pesquisas relacionadas aos adenocarcinomas em condições in vivo e in vitro.

Questões éticas ainda são debatidas em relação ao sistema CRISPR-Cas9, mas diante do diagnóstico e da dificuldade do tratamento do câncer de pulmão, o sistema CRISPR-Cas9 pode ser uma das principais formas de desenvolver tratamentos contra o câncer pulmonar e utilizar da terapia gênica como um meio para tal objetivo, pois esta possui diferentes formas de ser utilizada. Seja através de células de defesa, oncogenes ou genes supressores de tumor.

Com o desenvolvimento de pesquisa utilizando o sistema CRISPR-Cas9 e a evolução da tecnologia, o futuro do tratamento de diversas patologias, principalmente do câncer, passa a ser bastante promissor. Tornando do mecanismo imunológico de uma bactéria, o tratamento de diversas doenças, além da sua utilização em outras áreas que são de grande necessidade da humanidade.

\section{Referências}

1. Forman D, Mathers C, Soerjomataram I, Bray F, Eser S, Rebelo $M$, et al. Cancer incidence and mortality worldwide: Sources, methods and major patterns in GLOBOCAN 2012. Int. J. Cancer. 2014;136(5):359-86.

2. Abrahão KDS. Ministério da Saúde Instituto Nacional de Câncer. 2a. Rio de Janeiro, RJ: INCA; 2012.

3. Instituto Nacional do Câncer. Estimativa 2018. Incidencia de cáncer no Brasil. 2017.

4. Boch J. news and views TALEs of genome targeting A mucosal gateway for vaccines. Nat. Biotechnol. 2011;29(2):135-6.

5. Szczepek M, Brondani V, Büchel J, Serrano L, Segal DJ, Cathomen T. Structure-based redesign of the dimerization interface reduces the toxicity of zinc-finger nucleases. Nat. Biotechnol. 2007;25(7):786-93.

6. Makino K, Nakata A, Ishino Y, Amemura M, Shinagawa H. Nucleotide sequence of the iap gene, responsible for alkaline phosphatase isozyme conversion in Escherichia coli, and identification of the gene product. J. Bacteriol. 1987;169(12):5429-33. 
7. Barrangou R, Horvath P. CRISPR/Cas, the Immune System of Bacteria and Archaea. Science (80). 2010;327(5962):167-70.

8. Marraffini LA, Sontheimer EJ. CRISPR interference: RNAdirected adaptive immunity in bacteria and archaea. Nat. Rev. Genet. 2010;11(3):181-90.

9. Makarova KS, Brouns SJJ, Horvath P, Sas DF, Wolf YI, Yakunin $A F$, et al. Evolution and classification of the CRISPR-Cas systems. Nat. Rev. Microbiol. 2011;9(6):467-77.

10. Zhang F, Wen Y, Guo X. CRISPR/Cas9 for genome editing: Progress, implications and challenges. Hum. Mol. Genet. 2014;23(R1):40-6.

11. Ginn SL, Alexander IE, Edelstein ML, Abedi MR, Wixon J. Gene therapy clinical trials worldwide to 2012 - an update. J. Gene Med. 2013;15(January):65-77.

12. Pahle J, Walther W. Vectors and strategies for nonviral cancer gene therapy. Expert Opin. Biol. Ther. 2015;16(4):443-61.

13. Gonçalves GAR, Paiva R de MA. Gene therapy: advances, challenges and perspectives. Einstein (São Paulo). 2017;15(3):369-75. 14. Nichol G, Lee G, June CH, Holmes MC, Binder-Scholl G, Kalos $M$, et al. Gene Editing of CCR5 in Autologous CD4 T Cells of Persons Infected with HIV. N. Engl. J. Med. 2014;370(10):901-10. 15. Karimian A, Azizian K, Parsian H, Rafieian S, Shafiei-IrannejadV, Kheyrollah M, et al.CRISPR/Cas9 technology as a potent molecular tool for gene therapy. J. Cell. Physiol. 2019;234(8):12267-77.

16. Makarova KS, Wolf YI, Alkhnbashi OS, Costa F, Shah SA, Saunders SJ, et al. An updated evolutionary classification of CRISPR-Cas systems. Nat. Rev. Microbiol. 2015;13(11):722-36.

17. Koonin E V., Makarova KS, Zhang F. Diversity, classification and evolution of CRISPR-Cas systems. Curr. Opin. Microbiol. 2017;37:67-78.

18. Charpentier E. CRISPR-Cas9: how research on a bacterial RNAguided mechanism opened new perspectives in biotechnology and biomedicine. EMBO Mol. Med. 2015;7(4):363-5.

19. Wei Y, Terns RM, Terns MP. Cas 9 function and host genome sampling in type II-A CRISPR-cas adaptation. Genes Dev. 2015;29(4):356-61.

20. Oost JVan Der, Jore MM, Westra ER, Lundgren M, Brouns SJJ. CRISPR-based adaptive and heritable immunity in prokaryotes. 2009;(July):401-7.

21. Darband SG, Mirza-Aghazadeh-Attari M, Kaviani M, Mihanfar A, Sadighparvar S, Yousefi B, et al. Exosomes: natural nanoparticles as bio shuttles for RNAi delivery. J. Control. Release. 2018;289:158-70.

22. Kaushik I, Ramachandran S, Srivastava SK. CRISPR-Cas9: A multifaceted therapeutic strategy for cancer treatment. Semin. Cell Dev. Biol. 2019;(January):in press.

23. Hsu PD, Lander ES, Zhang F. Development and applications of CRISPR-Cas9 for genome engineering. Cell. 2014;157(6):1262-78.
24. Schmidt F, Grimm D. CRISPR genome engineering and viral gene delivery: A case of mutual attraction. Biotechnol. J. 2015;258-72.

25. Xiao-Jie L, Hui-Ying X, Zun-Ping K, Jin-Lian C, Li-Juan J. CRISPR-Cas9: A new and promising player in gene therapy. J. Med. Genet. 2015;52(5):289-96.

26. Nair J, Nair A, Veerappan S, Sen D. Translatable gene therapy for lung cancer using Crispr CAS9-an exploratory review. Cancer Gene Ther. 2019;1.

27. Katz A, Araujo LH, Baldotto C, Castro Jr G de, Gil Ferreira C, Mathias $C$, et al. Câncer de pulmão no Brasil. J. Bras. Pneumol. 2018;44(1)(1):55-64.

28. Travis WD, Brambilla E, Nicholson AG, Yatabe $Y$, Austin JHM, Beasley MB, et al. The 2015 World Health Organization Classification of Lung Tumors: Impact of Genetic, Clinical and Radiologic Advances since the 2004 Classification. J. Thorac. Oncol. 2015;10(9):1243-60.

29. Vansteenkiste J, Crino L, Dooms C, Douillard JY, Lim E, Rocco $\mathrm{G}$, et al. Annals of Oncology Advance Access published February 22, 2014 Key Message : "This manuscript provides recommendations for the diagnosis, treatment and follow $\nabla$ up of early stage non $\otimes$ small cell lung cancer developed during the 2 nd ESMO Consensus Confe. 2014;1-37.

30. Curran WJ, Hirsch FR, Wu Y-L, Mulshine JL, Scagliotti G V, PazAres $L$, et al. Lung cancer: current therapies and new targeted treatments. Lancet. 2016;389(10066):299-311.

31. Ministério da saúde. Sistema De Informação Ambulatoriais Do Sus. 2006;1-110.

32. Alexandrov LB, Nik-Zainal S, Wedge DC, Aparicio SAJR, Sam Behjati AVB, Bignell GR, et al. Signatures of mutational processes in human cancer. Nat. 500. 2013;415-21.

33. Hanahan D, Weinberg RA. Hallmarks of cancer: The next generation. Cell. 2011;144(5):646-74.

34. Jiang C, Meng L, Yang B, Luo X. Application of CRISPR/Cas9 gene editing technique in the study of cancer treatment. Clin. Genet. 2019;(June):1-16.

35. Eichner LJ, Brun SN, Herzig S, Young NP, Curtis SD, Shackelford DB, et al. Genetic Analysis Reveals AMPK Is Required to Support Tumor Growth in Murine Kras-Dependent Lung Cancer Models. Cell Metab. 2019;29(2):1-18.

36. Platt RJ, Chen S, Zhou Y, Yim MJ, Swiech L, Kempton HR, et al. CRISPR-Cas9 knockin mice for genome editing and cancer modeling. Cell. 2014;159(2):440-55.

37. Mali P, Esvelt KM, Church GM. Cas9 as a versatile tool for engineering biology. Nat. Methods. 2013;10(10):957-63.

38. Yi L, Li J. Biochimica et Biophysica Acta CRISPR-Cas9 therapeutics in cancer : promising strategies and present challenges. BBA - Rev. Cancer. 2016;1866(2):197-207. 
39. Makarova KS, Wolf YI, Koonin E V. Classification and Nomenclature of CRISPR-Cas Systems: Where from Here? Cris. J. 2018;1(5):325-36.

40. Knight SB, Crosbie PA, Balata H, Chudziak J, Hussell T, Dive C. Progress and prospects of early detection in lung cancer. Open Biol. 2017;7(9):1-12.

41. Moses C, Nugent F, Waryah CB, Garcia-Bloj B, Harvey AR, Blancafort P. Activating PTEN Tumor Suppressor Expression with the CRISPR/dCas9 System. Mol. Ther. - Nucleic Acids. 2019;14(March):287-300.

42. Oliveira BT De, Reis ÉVB. CRISPR-CAS9, Biossegurança e bioética: uma análise jusfilosófica-ambiental da engenharia genética. Veredas do Direito. 2019;16:123-52. 